\title{
Phosphodiesterase 4 regulates the migration of B16-F10 melanoma cells
}

\author{
YOSHIHIRO WATANABE, TAKU MURATA, KASUMI SHIMIZU, HIROSHI MORITA, \\ MADOKA INUI and TOSHIRO TAGAWA
}

\begin{abstract}
Department of Oral and Maxillofacial Surgery, Division of Reparative and Regenerative Medicine, Institute of Medical Science, Mie University Graduate School of Medicine, Tsu, Mie 514-8507, Japan
\end{abstract}

Received March 23, 2012; Accepted May 14, 2012

DOI: $10.3892 /$ etm.2012.587

\begin{abstract}
Phosphodiesterases (PDEs) are important regulators of signal transduction processes. Eleven PDE gene families (PDE1-11) have been identified and several PDE isoforms are selectively expressed in various cell types. PDE4 family members specifically hydrolyze cyclic AMP (cAMP). Four genes (PDE4A-D) are known to encode PDE4 enzymes, with additional diversity generated by the use of alternative mRNA splicing and the use of different promoters. While PDE4 selective inhibitors show therapeutic potential for treating major diseases such as asthma and chronic obstructive pulmonary disease, little is known concerning the role of PDE4 in malignant melanoma. In this study, we examined the role of PDE4 in mouse B16-F10 melanoma cells. In these cells, PDE4 activity was found to be $\sim 60 \%$ of total PDE activity. RT-PCR detected only PDE4B and PDE4D mRNA. Cell growth was inhibited by the cAMP analog, 8-bromo-cAMP, but not by the specific PDE4 inhibitors, rolipram and denbufylline, which increased intracellular cAMP concentrations. Finally, migration of the B16-F10 cells was inhibited by the PDE4 inhibitors and 8-bromo-cAMP, while migration was increased by a protein kinase A (PKA) inhibitor, $\mathrm{PKI}_{14-22}$, and was not affected by 8-pCPT-2'-O-Me-cAMP, which is an analog of exchange protein activated by cAMP (Epac). The inhibitory effect of rolipram on migration was reversed by $\mathrm{PKI}_{14-22}$. Based on these results, PDE4 appears to play an important role in the migration of B16-F10 cells, and therefore may be a novel target for the treatment of malignant melanoma.
\end{abstract}

Correspondence to: Dr Yoshihiro Watanabe, Department of Oral and Maxillofacial Surgery, Division of Reparative and Regenerative Medicine, Institute of Medical Science, Mie University Graduate School of Medicine, 2-174 Edobashi, Tsu, Mie 514-8507, Japan E-mail: yosi1017@clin.medic.mie-u.ac.jp

Key words: migration, B16-F10 melanoma cells, cyclic AMP, phosphodiesterase 4

\section{Introduction}

Melanoma is a major public health problem in many countries. Effective treatment depends upon early diagnosis followed by surgical excision with adequately wide margins. Medical therapies have contributed little to enhancing the control of established metastatic disease (1). Over the past 40 years no drug, or combination of drugs, has shown any impact on the survival of patients with metastatic melanoma; however, molecular pathways have been recently identified that are central to melanoma growth and apoptosis. These pathways are now under intense investigation as potential therapeutic targets $(1,2)$.

The cyclic nucleotide phosphodiesterase (PDE) superfamily represents 11 gene families (PDE1-11) which differ in their biochemical properties, regulation and sensitivity to pharmacological agents. PDEs regulate signal transduction by modulating intracellular levels of cyclic AMP (cAMP) and cGMP. Three of the 11 PDE families (PDE4, PDE7 and PDE8) selectively hydrolyze cAMP, 3 families (PDE5, PDE6 and PDE9) selectively hydrolyze cGMP and 5 families (PDE1, PDE2, PDE3, PDE10 and PDE11) hydrolyze both cyclic nucleotides (3).

PDE4 family members, which exclusively hydrolyze cAMP, are widely expressed and play major regulatory roles. Much of the functional knowledge of PDE4 enzymes was deduced from experiments using highly selective inhibitors, targeted gene knockout, small inhibitory RNA-mediated ablations or dominant-negative mutations to disrupt endogenous intracellular enzyme activities (4). Four genes (PDE4A, PDE4B, PDE4C and PDE4D) encode more than 20 distinct PDE4 isoforms as a result of mRNA splicing and the use of distinct promoters $(5,6)$. The various isoforms encoded by a single gene can differ in their enzymatic properties and tissue distributions. Furthermore, these diverse isoforms each interact with various distinct scaffolding proteins, leading to different roles in compartmentalized intracellular signaling. The PDE4 family has attracted considerable attention over the past decade as its selective inhibitors have potential therapeutic use for a wide range of major disease areas such as asthma, chronic obstructive pulmonary disease, depression and Parkinson's disease (4).

It is suggested that PDE4 is expressed in several types of malignant tumor cells, where this family may induce apoptosis (7) or regulate cell migration (8). However, the expression patterns and roles of PDE4 in malignant melanoma cells are 
still unclear. In this study, we examined expression patterns and roles of PDE4 in mouse B16-F10 melanoma cells.

\section{Materials and methods}

Cell culture. B16-F10 cells, a highly metastatic lung selected cell subline derived from C57/BL6 murine melanoma, are frequently used for the migration experiments (9). B16-F10 cells (American Type Culture Collection, Manassas, VA, USA) were maintained in Dulbecco's modified Eagle's medium (DMEM) containing 10\% fetal bovine serum (FBS) (Invitrogen Life Technologies, Carlsbad, CA, USA) at $37^{\circ} \mathrm{C}$ in a humidified $5 \% \mathrm{CO}_{2}$ atmosphere. The medium was changed 3 times/week.

Preparation of cell extracts for cAMP PDE assay. B16-F10 cells were seeded at $2.5 \times 10^{5}$ cells $/ 25 \mathrm{~cm}^{2}$ flask. After 3 days, the cells were washed twice with phosphate buffered saline (PBS), harvested with a rubber policeman and then homogenized in $1 \mathrm{ml}$ of ice-cold homogenization buffer (100 mM TES, pH 7.4, $10 \mu \mathrm{g} / \mathrm{ml}$ each of pepstatin, leupeptin and aprotinin, $1 \mathrm{mM}$ benzamidine, $0.5 \mathrm{mM}$ Pefabloc, $1 \mathrm{mM}$ EDTA, $0.1 \mathrm{mM}$ EGTA, $5 \mathrm{mM} \mathrm{MgSO}_{4}$ and $10 \%$ glycerol).

cAMP PDE assay. cAMP PDE activity was assayed as previously described (10). Samples were incubated at $30^{\circ} \mathrm{C}$ for $10 \mathrm{~min}$ in a total volume of $0.3 \mathrm{ml}$, containing $50 \mathrm{mM}$ HEPES, pH 7.4, $0.1 \mathrm{mM}$ EGTA, $8.3 \mathrm{mM} \mathrm{MgCl}{ }_{2}$ and $0.1 \mu \mathrm{M}\left[{ }^{3} \mathrm{H}\right] \mathrm{cAMP}$ $(18,000 \mathrm{cpm})$. PDE 4 activity was measured as the cAMP PDE activity inhibited by $10 \mu \mathrm{M}$ rolipram, a specific inhibitor of PDE4 (11).

$R T$-PCR. B16-F10 cells were seeded at $2.5 \times 10^{5}$ cells $/ 25 \mathrm{~cm}^{2}$ flask. After 3 days, total RNA was isolated from B16-F10 cells using the QuickGene RNA cultured cell kit $\mathrm{S}$, and from mouse heart using the QuickGene RNA tissue kit S (Fuji Photo Film Co., Tokyo, Japan). Mouse kidney total RNA was purchased from Stratagene (Santa Clara, CA, USA). First-strand cDNA was generated from total RNA using a High Capacity RNA-to$\mathrm{cDNA}^{\mathrm{TM}}$ kit (Applied Biosystems, Foster City, CA, USA). PCR was performed with specific oligonucleotide primer sets for PDE4A, PDE4B, PDE4C, PDE4D, PDE4B2 and PDE4D5 (Table I). The PCR reaction was carried out in a total volume of $50 \mu \mathrm{l}$ containing $10 \mathrm{mM}$ Tris- $\mathrm{HCl}(\mathrm{pH} 8.3), 50 \mathrm{mM} \mathrm{KCl}$, $1.5 \mathrm{mM} \mathrm{MgCl}_{2}, 200 \mu \mathrm{M}$ dNTPs, 2.5 units HotStarTaq ${ }^{\mathrm{TM}}$ DNA Polymerase (Qiagen, Hilden, Germany) and $10 \mu \mathrm{M}$ sense and antisense primers. HotStarTaq ${ }^{\mathrm{TM}}$ DNA Polymerase was activated by incubation of the reactions at $95^{\circ} \mathrm{C}$ for $15 \mathrm{~min}$. For PDE4A, PDE4B, PDE4C, PDE4D and PDE4B2, this activation step was followed by 30 cycles of amplification $\left(94^{\circ} \mathrm{C}\right.$ for $1 \mathrm{~min}, 62^{\circ} \mathrm{C}$ for $1 \mathrm{~min}$ and $72^{\circ} \mathrm{C}$ for $1 \mathrm{~min}$ ) and $72^{\circ} \mathrm{C}$ for $10 \mathrm{~min}$. For PDE4D5, this was followed by 30 cycles of amplification $\left(94^{\circ} \mathrm{C}\right.$ for $30 \mathrm{sec}, 60^{\circ} \mathrm{C}$ for $30 \mathrm{sec}$ and $72^{\circ} \mathrm{C}$ for $30 \mathrm{sec}$ ) and $72^{\circ} \mathrm{C}$ for $7 \mathrm{~min}$. Products were subjected to electrophoresis on $2 \%$ agarose gels and visualized by a SYBR-Green nucleic acid gel stain (Molecular Probes, Inc., Eugene, OR, USA).

Growth experiments. The cells were plated in a 96-well plate (100 cells/well) and allowed to adhere for $24 \mathrm{~h}$. The cells were then cultured in the absence or presence of different concentrations of reagents for 6 days. MTS [3-(4,5-dimethyl-
Table I. Primer sequences used for RT-PCR.

\begin{tabular}{llc}
\hline & \multicolumn{1}{c}{ Primer } & $\begin{array}{c}\text { Product } \\
\text { size (bp) }\end{array}$ \\
\hline PDE4A & 5'-TTCAAGCTGCTGCAAGAAGA-3' & 225 \\
& 5'-TTCCTGAGGACCTGGATACG-3' & \\
PDE4B & 5'-GAACAAATGGGGCCTTAACA-3' & 609 \\
& 5'-TTGTCCAGGAGGAGAACACC-3' & \\
PDE4C & 5'-CATGCTCAACCGTGAGTTGT-3' & 372 \\
& 5'-TGGAACGTCTTGAGGAGGTC-3' & \\
PDE4D & 5'-GGAGCTTGTCACCTTCTTGG-3' & 181 \\
& 5'-GTGGGCTTTAAGTTGCTCCA-3' & \\
PDE4B2 & 5'-GAGCACCAGAAAGAGCTTGG-3' & 345 \\
& 5'-CAGACACCTGGTTCCCTGAT-3' & \\
PDE4D5 & 5'-ATCCGTTTCTCCCAAGCTCT-3' & 365 \\
& 5'-CAGGCTAGCCAAGACCTGAG-3' & \\
\hline
\end{tabular}

thiazol-2-yl)-5-(3-carboxymethoxyphenyl)-2-(4-sulfophenyl)$2 \mathrm{H}$-tetrazolium, inner salt] assays were performed using a CellTiter $96^{\circledR}$ Aqueous One Solution cell proliferation assay (Promega, Madison, WI, USA) and cell numbers were calculated.

cAMP concentration in mouse B16-F10 melanoma cells. B16-F10 cells were plated in a 6 -well plate $\left(3 \times 10^{5}\right.$ cells/well). After a 48-h incubation, the cells were incubated with medium containing the indicated concentrations of reagents for $30 \mathrm{~min}$. Intracellular cAMP content was determined using a Cyclic AMP EIA kit (Cayman Chemical Co., Inc., Ann Arbor, MI, USA).

Migration assay. Cell migration was assayed as previously described (12). Briefly, cells $\left(2.5 \times 10^{4}\right.$ cells in DMEM medium containing $0.1 \%$ FBS with the indicated concentrations of reagents) were transferred to $8-\mu \mathrm{m}$ pore BD Falcon ${ }^{\mathrm{TM}}$ cell culture inserts (BD Biosciences, Bedford, MA, USA). The inserts, which were coated with fibronectin $(6 \mu \mathrm{g} / \mathrm{membrane})$ on the lower surface of the membrane, were placed in companion wells containing DMEM medium supplemented with $20 \%$ FBS as a chemoattractant. Following a 12-h incubation, the inserts were removed and the cells on the upper surface of the membranes were wiped with a cotton swab. Cells on the lower surface of the membrane were then fixed and stained with Diff-Quick Stain ${ }^{\mathrm{TM}}$ (Sysmex Co., Ltd., Kobe, Japan). The cells on the membrane were then counted under a microscope.

Statistical analysis. Differences were statistically analyzed using the Tukey-Kramer multiple comparisons test or Student's t-test (Fig. 5C). Significance was defined as a calculated P-value $<0.05$.

\section{Results}

PDE4 activity in B16-F10 cells. To test whether PDE4 is expressed in B16-F10 cells, we used the PDE4-specific 


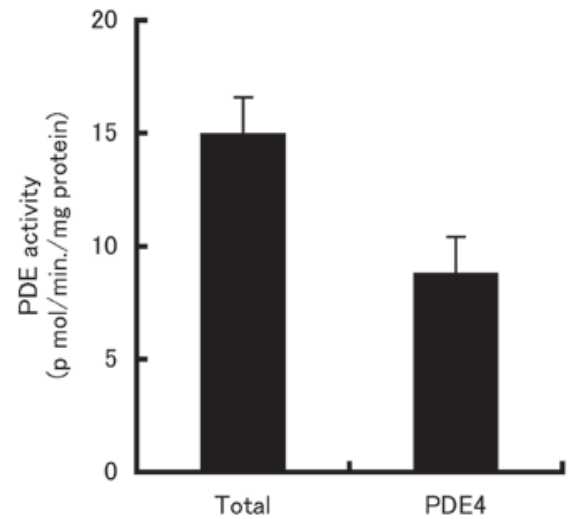

Figure 1. PDE4 activity in B16-F10 cells. Homogenates were prepared and assayed for PDE activity with or without $10 \mu \mathrm{M}$ rolipram as described in the materials and methods. The error bars represent the mean $\pm \mathrm{SD}$ of three different experiments.

A

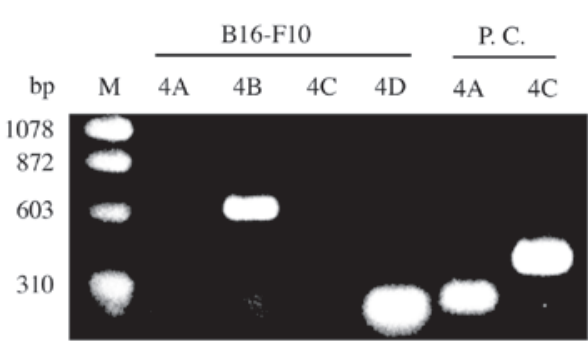

B

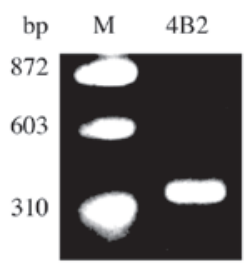

$\mathrm{C}$

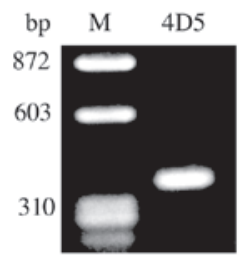

Figure 2. mRNA expression of PDE4 isoforms in B16-F10 cells as determined by RT-PCR. (A) Expression of PDE4 mRNAs in B16-F10 cells. Molecular markers (M) and B16-F10 cell PDE4s (B16-F10): PDE4A (4A), PDE4B (4B), PDE4C (4C) and PDE4D (4D). Positive controls (P.C.) were also used: mouse heart PDE4A (4A) and mouse kidney PDE4C (4C). (B and C) Expression of PDE4B2 and PDE4D5 splice variant mRNAs in B16-F10 cells. Molecula markers (M), B16-F10 cell PDE4B2 (4B2) and PDE4D5 (4D5) are indicated.

inhibitor, rolipram. As shown in Fig. 1, 60\% of the total PDE activity in extracts of B16-F10 cells was PDE4 activity.

PDE4 mRNA expression in B16-F10 cells. To determine which PDE4 isoforms (PDE4A, PDE4B, PDE4C and/or PDE4D) are expressed in B16-F10 cells, we performed RT-PCR. PDE4B and PDE4D, but not PDE4A or PDE4C, mRNA were detected by RT-PCR in total RNA from B16-F10 cells (Fig. 2A). Over 20 different PDE4 isoforms are generated from 4 genes through
A

B
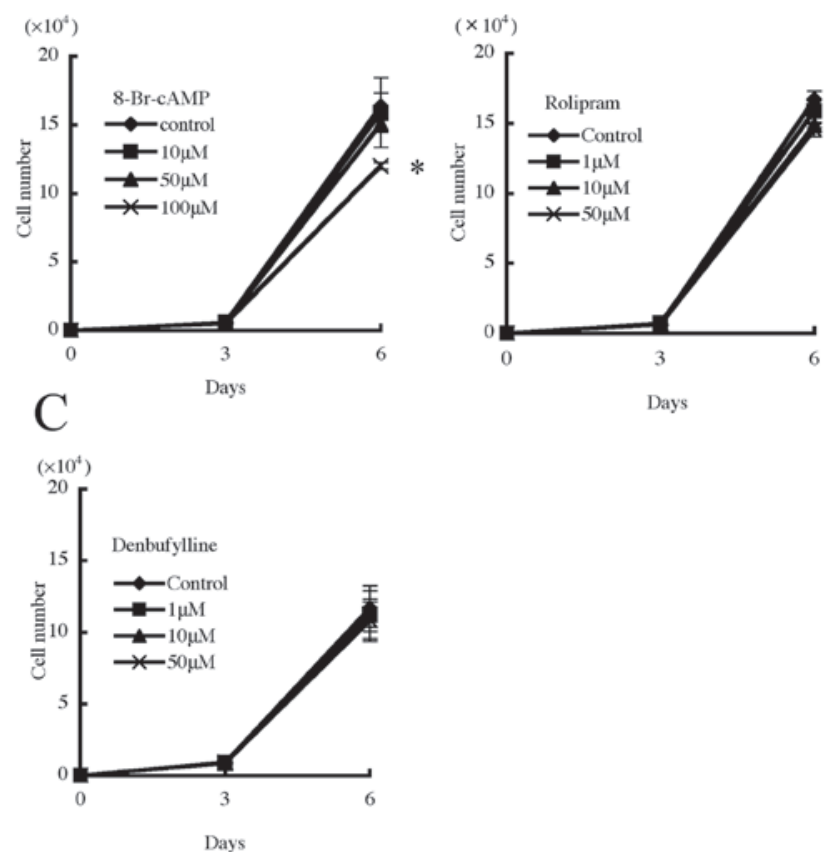

$\mathrm{D}$
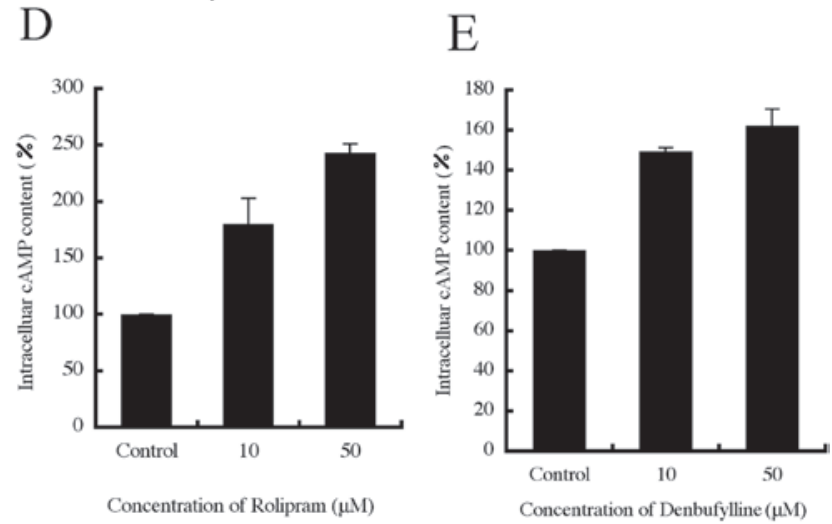

Figure 3. PDE4 inhibitors do not affect B16-F10 cell growth. Cells were plated in 96-well plates and cultured with different concentrations as indicated of the following reagents: (A) 8-Br-cAMP, (B) rolipram and (C) denbufylline. The error bars represent the mean \pm SD of three different experiments. The treatments that differed significantly from the control are noted ("P<0.05). Effects of (D) rolipram and (E) denbufylline on intracellular cAMP concentration. cAMP concentration was measured as described in Materials and methods. Values represent the mean \pm one-half of the range of two independent experiments.

the use of specific promoters and alternative mRNA splicing. Interestingly, we were able to detect PDE4B2 and PDE4D5 mRNA in the RNA from B16-F10 cells (Fig. 2B and C).

Effect of a cAMP analog and PDE4-specific inhibitors on B16-F10 cell growth. While the cAMP analog, 8-bromo-cAMP (8-Br-cAMP), suppressed cell growth after 6 days of treatment (Fig. 3A), no effects were observed after treatment with the PDE4-specific inhibitors rolipram and denbufylline (Fig. 3B and $\mathrm{C}$ ). To verify the efficacy of rolipram and denbufylline, we next measured cAMP concentration in the treated cells. After a 30-min treatment, the intracellular cAMP concentration was significantly increased by rolipram and denbufylline at concentrations of 10 and $50 \mu \mathrm{M}$ for both drugs (Fig. 3D and E). 
A

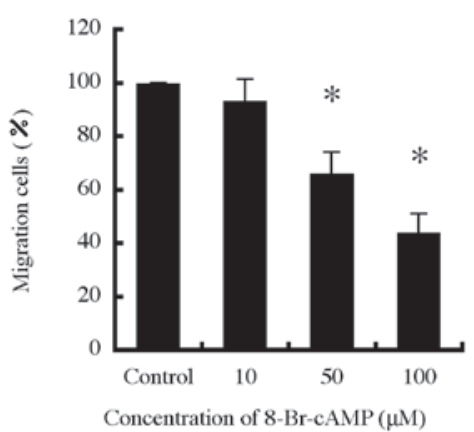

B

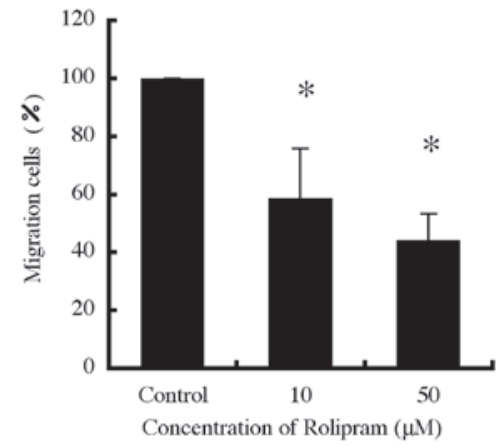

C

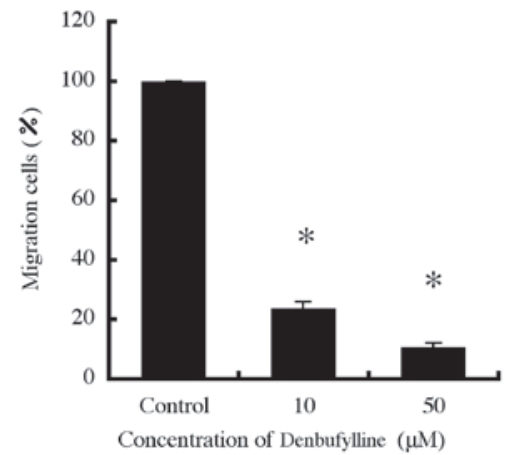

Figure 4. Role of PDE4 in B16-F10 cell migration. B16-F10 cells were transferred to inserts for migration assays as indicated in Materials and methods. Inserts were incubated with different concentrations of the reagents: (A) 8-Br-cAMP, (B) rolipram and (C) denbufylline. The cells were counted as described in Materials and methods. The error bars represent the mean \pm SD of three different experiments. The treatments that differed significantly from the control are noted $\left({ }^{*} \mathrm{P}<0.05\right)$.

These results confirm that rolipram and denbufylline suppress PDE4s and increase intracellular cAMP concentration.

Effect of a cAMP analog and PDE4-specific inhibitors on B16-F10 cell migration. We next investigated whether PDE4 is necessary for B16-F10 cell migration. We found that 8-Br-cAMP inhibited B16-F10 cell migration (Fig. 4A). Interestingly, the PDE4-specific inhibitors, rolipram and denbufylline inhibited migration in a dose-dependent manner (Fig. 4B and C). It was confirmed that there were no significant changes in the number of cells after having been cultured with each agent (data not shown).

Effect of PKA and Epac on B16-F10 cell migration. Since 8-Br-cAMP activates both protein kinase A (PKA) and Epac (13), we decided to investigate whether targeting these proteins affects cell migration. We first used H-89, a widely used PKA
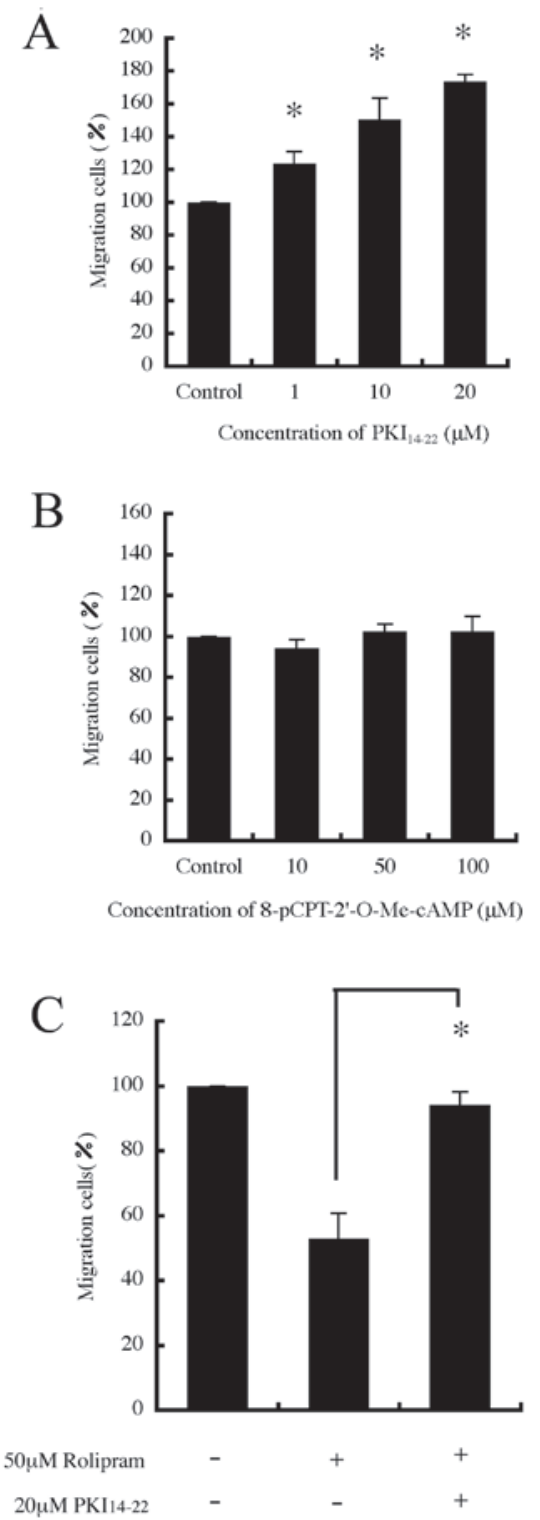

Figure 5. Downstream PKA signaling mediates the effects of PDE4 on B16-F10 cell migration. The cells were transferred to inserts for migration assays with indicated concentrations of the following reagents: (A) $\mathrm{PKI}_{14-22}$, (B) 8-pCPT-2'-O-Me-cAMP and (C) rolipram with or without $\mathrm{PKI}_{14-22}$. The cells were counted as described in Materials and methods. The error bars represent the mean $\pm \mathrm{SD}$ of three different experiments. (A and B) The treatments that differed significantly from the control are noted $\left({ }^{*} \mathrm{P}<0.05\right)$. $(\mathrm{C})$ Rolipram $(50 \mu \mathrm{M})$ plus $\operatorname{PKI}_{14-22}(20 \mu \mathrm{M})$ is compared with rolipram $(50 \mu \mathrm{M})$ without $\mathrm{PKI}_{14-22}\left({ }^{*} \mathrm{P}<0.05\right)$.

inhibitor, and found that $\mathrm{H}-89$ promoted cell migration in a dose-dependent manner (data not shown). However, H-89 inhibits other protein kinases at concentrations similar to those affecting PKA (14). Therefore, we also studied the effect of a PKA inhibitor, $\mathrm{PKI}_{14-22}$, and found that $\mathrm{PKI}_{14-22}$ also promoted cell migration in a dose-dependent manner (Fig. 5A). In contrast, an Epac activator, 8-pCPT-2'-O-Me-cAMP that only activates Epac without any concomitant activation of PKA on cell migration, had no effect (Fig. 5B). Furthermore, the inhibitory effect of rolipram on cell migration was reversed by $\mathrm{PKI}_{14-22}$ (Fig. 5C). It was confirmed that there were no significant changes in the number of cells after having been cultured with each agent (data not shown). 


\section{Discussion}

Surgical resection of a malignant melanoma provides an effective first-line therapy; however, melanoma can progress to an aggressive, metastasizing form of the disease with extremely poor patient survival rates (15). Induction of apoptosis by increasing cAMP levels with PDE4 inhibitors has been demonstrated for colon cancer and chronic lymphoid leukemia, suggesting that PDE4 inhibitors, in clinical development for different therapeutic indications, could be a new strategy for cancer treatment (16). Although previous studies report the expression of PDEs in melanoma, their contribution to tumor pathology remains obscure (17). Previous studies have demonstrated that proliferation in B16-F10 cells (a highly metastatic lung selected subline derived from murine melanoma) is suppressed and cell morphology is altered by pentoxifylline, a well-known nonspecific PDE inhibitor with the possible involvement of cAMP-PKA signaling (18). However, the study failed to determine which PDEs were involved. Here, we found that the main cAMP-PDE activity in extracts of B16-F10 cells involved PDE4, and we detected both PDE4B and PDE4D mRNA by RT-PCR. PDE4 isoforms have a role in achieving compartmentalized signaling in cells by interacting with various scaffold proteins. PDE4B and PDE4D have been reported to have distinct patterns of distribution in mouse embryonic fibroblasts and control cAMP in different cellular subdomains (19). These two isoforms might be play different roles in B16-F10 cells. Over 20 different isoforms are generated from 4 genes through the use of specific promoters and alternative mRNA splicing. Recently, Marquette et al (16) reported that PDE4B2 and PDE4D5 are expressed in human melanoma cell lines, whereas mouse melanocytes express only PDE4B2 and not PDE4D. We also detected PDE4B2 and PDE4D5 mRNA in B16-F10 cells. These data suggest that PDE4D5 may be important in malignant melanoma.

Although 8-Br-cAMP suppressed B16-F10 cell proliferation, PDE4 specific inhibitors did not show any effects on proliferation. The PDE4 inhibitors were functional as they yielded an elevated cAMP concentration. Abusnina et al (20), reported that treatment with $10 \mu \mathrm{M}$ rolipram for $24 \mathrm{~h}$ had no effect on B16-F10 cell proliferation, which is consistent with our results. We also tested the effect of rolipram on proliferation for 6 days, but no change was observed. It was suggested that either PDEs may form an enzymatic barrier to cAMP diffusion or, alternatively, compartmentalized PDEs may act as a sink to localized pools of cAMP (19). Therefore, it is probable that other PDEs are involved in proliferation. In B16-F10 cells, in addition to PDE4, PDE8 activity was detected (20) as cAMPPDE, and by RT-PCR, we detected PDE1A, PDE1B, PDE1C, PDE7A, PDE10A and PDE11A (data not shown).

Notably, PDE4-specific inhibitors suppress B16-F10 cell migration. Recent research demonstrated that RhoA and Rac1 GTPases induce B16-F10 cell motility (21). PKA, which is a major effector of cAMP, induces phosphorylation of RhoA and regulates its function in several cell types (22). It is also reported that PKA-induced phosphorylation of RhoA, as a consequence of PDE4 inhibition, provides a probable mechanism for the reduced RhoA-GTP levels and subsequent effects on peripheral microspike formation noted in rat embryo fibroblast REF52 cells (22). We also found that $\mathrm{PKI}_{14-22}$ promoted cell migration. To investigate whether the inhibitory effect of PDE4 inhibition on B16-F10 motility is mediated by enhanced PKA activation, we also tested the effects of $\mathrm{PKI}_{14-22}$ on rolipram-mediated inhibition of migration. $\mathrm{PKI}_{14-22}$ reversed rolipram-mediated inhibition by almost $100 \%$. Furthermore, we used another effector of cAMP, Epac, which serves as GEFs for the small GTPases Rap1 and Rap2 (22). In contrast to the effects seen with PKA inhibition, 8-pCPT-2'-O-Me-cAMP had no effect on migration. This suggests that PDE4 inhibition suppresses B16-F10 cell migration by inducing downstream PKA activation. Furthermore, Serrels et al (8) showed that PDE4D5 regulates chemically induced squamous cell carcinoma cell polarity. However, in REF 52 cells, it is unclear which PDE4 isoform(s) regulates microspike formation (22). PDE4 isoform(s) that function to regulate cell migration may differ in different cell types. More studies are required to determine which isoform(s), regulate cell migration in a wider panel of cell lines.

In conclusion, our results suggest that PDE4 regulates cell migration via cAMP-PKA signaling in B16-F10 cells and that cAMP-PDE4 signaling may be a new therapeutic target for melanoma.

\section{Acknowledgements}

This study was supported by a grant from the Okasan-Kato Foundation to Y. Watanabe and a Grant-in-Aid for Scientific Research (B) from the Japan Society for the Promotion of Science (18390537) to T. Murata.

\section{References}

1. Thompson JF, Scolyer RA and Kefford RF: Cutaneous melanoma. Lancet 36: 687-701, 2005.

2. Eggermont AM and Robert C: New drugs in melanoma: it's a whole new world. Eur J Cancer 47: 2150-2157, 2011.

3. Beavo JA, Houslay MD and Francis SH: Cyclic nucleotide phosphodiesterase superfamily. In: Cyclic Nucleotide Phosphodiesterases In Health and Disease. Beavo JA, Francis SH, Hously MD (eds). CRC Press, New York, pp3-17, 2007.

4. Hously MD, Schafer P and Zhang KY: Keynote review: phosphodiesterase-4 as a therapeutic target. Drug Discov Today 10: 1503-1519, 2005.

5. Hously MD: Underpinning compartmentalized cAMP signaling through targeted cAMP breakdown. Trends Biochem Sci 35: 91-100, 2010 .

6. Bolger GB, Conti M and Houslay MD: Cellular function of PDE4 enzymes. In: Cyclic Nucleotide Phosphodiesterases In Health and Disease. Beavo JA, Francis SH, Hously MD (eds). CRC Press, New York, pp99-129, 2007.

7. Kim DH and Lerner A: Type 4 cyclic adenosine monophosphate phosphodiesterase as a therapeutic target in chronic lymphocytic leukemia. Blood 92: 2484-2494, 1998

8. Serrels B, Sandilands E, Serrels A, et al: A complex between FAK, RACK1, and PDE4D5 controls spreading initiation and cancer cell polarity. Curr Biol 20: 1086-1092, 2010.

9. Fidler IJ: Selection of successive tumour lines for metastasis. Nat New Biol 242: 148-149, 1973.

10. Murata T, Taira M and Manganiello VC: Differential expression of cGMP-inhibited cyclic nucleotide phosphodiesterases in human hepatoma cell lines. FEBS Lett 390: 29-33, 1996.

11. Houslay MD, Sullivan M and Bolger GB: The multienzyme PDE4 cyclic adenosine monophosphate-specific phosphodiesterase family: intracellular targeting, regulation, and selective inhibition by compounds exerting anti-inflammatory and antidepressant actions. Adv Pharmacol 44: 225-342, 1998.

12. Moutasim KA, Nystrom ML and Thomas GJ: Cell migration and invasion assays. Methods Mol Biol 731: 333-343, 2011. 
13. Holz GG, Chepumy OG and Schwede F: Epac-selective cAMP analogs: new tools with which to evaluate the signal transduction properties of cAMP-regulated guanine nucleotide exchange factors. Cell Signal 20: 10-20, 2008.

14. Davies SP, Reddy H, Caivano M and Cohen P: Specificity and mechanism of action of some commonly used protein kinase inhibitors. Biochem J 351: 95-105, 2000.

15. Houslay MD: Hard times for oncogenic BRAF-expressing melanoma cells. Cancer Cell 19: 3-4, 2011.

16. Marquette A, André J, Bagot M, Bensussan A and Dumaz N: ERK and PDE4 cooperate to induce RAF isoform switching in melanoma. Nat Struct Mol Biol 18: 584-591, 2011.

17. Lau $\mathrm{E}$ and Ronai Z: RAF-isotype switching: from $\mathrm{B}$ to $\mathrm{C}$ through PDE. Nat Struct Mol Biol 18: 517-518, 2011.

18. Dua P and Gude RP: Antiproliferative and antiproteolytic activity of pentoxifylline in cultures of B16F10 melanoma cells. Cancer Chemother Pharmacol 58: 195-202, 2006.
19. Blackman BE, Horner K, Heidmann J, et al: PDE4D and PDE4B function in distinct subcellular compartments in mouse embryonic fibroblasts. J Biol Chem 286: 12590-12601, 2011.

20. Abusnina A, Keravis T, Yougbare I, Bronner C and Lugnier C: Anti-proliferative effect of curcumin on melanoma cells is mediated by PDE1A inhibition that regulates the epigenetic integrator UHRF1. Mol Nutr Food Res 55: 1677-1689, 2011.

21. Dua P and Gude RP: Pentoxifylline impedes migration in B16F10 melanoma by modulating Rho GTPase activity and actin organisation. Eur J Cancer 44: 1587-1595, 2008.

22. Fleming YM, Frame MC and Houslay MD: PDE4-regulated cAMP degradation controls the assembly of integrin-dependent actin adhesion structures and REF52 cell migration. J Cell Sci 117: 2377-2388, 2004. 